\title{
A PROPOS DES COCCIDIES DES OISEAUX SAUVAGES
}

\author{
Par W.-L. YAKIMOFF et W.-F. GOUSSEFF
}

\section{Introduction}

Les oiseaux sauvages ont été très peu étudiés jusqu'ici au point de vue qui nous occupe, à l'exception toutefois des petits oiseaux (hirondelles, moineaux, alouettes, etc.), tous infectés soi-disant par Isospora lacazei Labbé, 1894. Peu de travaux sont relatifs au gibier (faisan, perdrix, gélinotte des bois, caille, etc.). Au sujet de ces derniers, nous avons des travaux sur les coccidies du faisan (Fantham, 1894 ; Sjörbing, 1897 ; Verwey, 1926), de la perdrix (Fantham, 1910 ; Brinkmann, 1926 ; Allen, 1934 ; Verwey, 1926), de la caille (Tyzzer, 1929 ; D. Henry, 1931) et du coq de bruyère (Galli-Valerio, 1927, 1929), « Berghuhn » (Yakimoff et Bouewitsch, 1932).

\section{Recherches personnelles}

En 1934, beaucoup d'oiseaux sauvages ont été examinés par nous en Russie Blanche et parmi eux 174 oiseaux différents dans deux districts : district de Poloztk, au Nord de la Russie Blanche (110 oiseaux) et district Gomel, au Sud (64 oiseaux). Ces oiseaux sont les suivants :

Hirondelle des champs (Chelidon rustica), hirondelle de ville (Delichon urbica), 1 Anthus trivialis, 1 Crex crex, 1 sansonnet (Sturnus vulgaris), 1 Querquedula frugilegus, 17 bécasses (Scolapax rusticola), 1 coq de bruyère (Lyrurus tetrix s. Tetrao tetrix), 25 tetras (Tetrao urogallus), 1 caprimilge (Caprimulgus europeus), 2 choucas (Colocus monedula), 1 passereau domestique (Passer domesticus), 33 merles chanteurs (Turdus philomelus), 3 Lynx torquilla, 12 corbeaux (Corvus cornix), 1 grue (Grus grus), 2 grands Dryobates major, 16 hiboux domestiques (Athene noctua), 3 perdrix blanches (Lagopus lagopus), 13 perdrix grises (Perdix perdix), 26 milans (Milvus migrans), 1 aigle (Hieraetus pennatus), 1 Lanius collurio, 2 Motacilla alba, 1 bruant (Emberiza citrinella), 2 Phonicurus phonicurus, 1 Coracias garrula, 1 Lachetes pugnax, 1 Chloris chloris, 1 Upupa epops, 1 Sitta europea, 1 pie (Pica pica) et 2 freux (Trypanocorax frugilegus). 
Parmi ces oiseaux les suivants se trouvaient être infectés par des coccidies : 1) coq de bruyère, 2) tetras, 3) Dryobates major, 4) perdrix, 5) moineau, 6) Crex crex, 7) Anthus trivialis, 8) hirondelle, 9) sansonnet, 10) Lynx torquilla, et 11) pie.

Sur 33 espèces d'oiseaux, 11 (33,3 p. 100) étaient infectés par les coccidies.

Ces coccidies appartiennent aux genres Eimeria et Isospora.

\section{Coccidies du coq de bruyère}

Nous examinâmes 25 oiseaux, dont $13(52$ p. 100) du district Poloztk avaient des coccidies des deux genres Isospora et Eimeria : dix oiseaux hébergeaient la première espèce, deux la seconde et un la première et la seconde.

\section{Première eśpèce}

Onze oiseaux en étaient infectés, dont un hébergeait la première espèce associée à la seconde. De nombreux oocystes se rencontraient chez 5 oiseaux et un petit nombre chez 6 .

La forme des oocystes est cylindrique; ils sont incolores; leur membrane est fort mince; le protoplasme occupe presque tout l'intérieur de l'oocyste.

La mensuration de 111 oocystes a donné les dimensions suivantes : 21,96-36,69 $\mu \times 12,20-19,52 \mu$, en moyenne $29,62 \mu \times 15,32 \mu$, les plus grands $36,60 \mu \times 15,56 \mu$, les plus petits $21,96 \mu \times 13,42 \mu$, le plus souvent $26,86 \mu \times 14,64 \mu$. Formindex : $1: 0,40-0,66$, en moyenne $1: 0,50$, le plus souvent $1: 0,50$.

Nous voyons que le formindex montre que, dans la plupart des cas, la longueur est égale à la largeur.

Des formes de développement montrèrent d'abord deux et ensuite quatre spores $(9,76 \mu \times 3,64 \mu)$. Dans les spores se développèrent deux sporozoïtes. Les sporocystes seuls renfermaient des reliquats ; les oocystes n'en avaient pas, mais les dernières avaient un granule polaire.

Cette Eimeria serait-elle une nouvelle espèce?

Galli-Valerio, en 1927, trouva chez le Lyrurus tetrix des oocystes de forme cylindrique, ressemblant à Eimeria sciurorum de l'écureuil. Le micropyle est peu développé. Les dimensions 24,0$27,0 \mu \times 15 \mu$. Le protoplasme, sphérique, présente de petits granules mesurant 9,7-12,0 $\mu$. La membrane est très mince. Quatre spores $(10,0 \mu \times 6,0 \mu)$, renfermant chacune deux sporozoïtes en forme 
de virgule. Pas de reliquat dans l'oocyste. L'auteur l'a dénommée Eimeria lyruri.

Il est possible que notre coccidie soit Eimeria lyruri.

\section{Deuxième espèce}

L'oocyste est complètement sphérique ou de forme sub-sphérique. Il y en avait beaucoup, sans micropyle et à membrane épaisse (1,5$2,0 \mu$ ).

Les dimensions des formes sphériques, mesurées au nombre de 49 , étaient les suivantes : 19,52-25,64 $\mu$, en moyenne $22,13 \mu$, le plus souvent $21,96 \mu$.

Les dimensions des formes sub-sphériques, mesurées au nombre de 50, étaient de : $20,74-29,27 \mu \times 17,08-24,40 \mu$, en moyenne $24,90 \mu \times 21,34 \mu$, les plus grandes $29,28 \mu \times 24,40 \mu$, les plus petites $20,74 \mu \times 18,30 \mu$, le plus souvent $24,40 \mu \times 20,74 \mu$. Formindex : $1: 0,77-0,95$, en moyenne $1: 0,86$, le plus souvent $1: 0,85$.

La sporulation donne quatre spores $(12,20 \mu \times 10,98 \mu)$ avec deux sporozoïtes dans chacune. Il n'y a point de reliquat dans l'oocyste ; il est difficile de dire s'il en existe dans les sporocystes. L'oocyste contient un granule polaire.

Galli-Valerio, en 1931, trouva chez le même oiseau une autre coccidie, Isospora lyruri. La forme des coccidies était sphérique ; les dimensions étaient de $15 \mu$, avec un léger promontoire à la place du micropyle, peu visible. Durant la sporulation se développent dans l'oocyste deux spores oviformes, mesurant $12,0 \mu \times 7,0 \mu$, avec quatre sporozoïtes $(6,0 \mu \times 3,0 \mu)$, sans le reliquat.

Nous croyons que cette coccidie est une nouvelle espèce et nous la nommons, en l'honneur du professeur G. A. Nadson, Eimeria nadsoni n. sp.

Vu la grande fréquence des coccidies chez le coq de bruyère, nous estimons que ces parasites sont les agents qui anéantissent ce magnifique gibier.

\section{Coccidies du tetras}

Nous avons examiné un tetras (district Polotzk) avec des coccidies des deux espèces.

\section{Première espèce}

La forme des oocystes est cylindrique. Leur couleur est jaunâtre; il n'y a pas de micropyle. Les dimensions, mesurées sur 55 oocystes, sont les suivantes: $21,96-34,16 \mu \times 14,64-18,30 \mu$, en moyenne $27,93 \mu \times 16,41 \mu$, les plus grandes $34,16 \mu \times 17,08 \mu$, les plus peti- 
tes $21,96 \mu \times 17,08 \mu$, le plus souvent $29,28 \mu \times 17,08 \mu$. Formindex : $1: 0,48-0,77$, en moyenne $1: 0,55$, le plus souvent $1: 0,53$ et $1: 0,58$. La sporulation donne quatre sporoblastes de $6,10 \mu$ et les spores

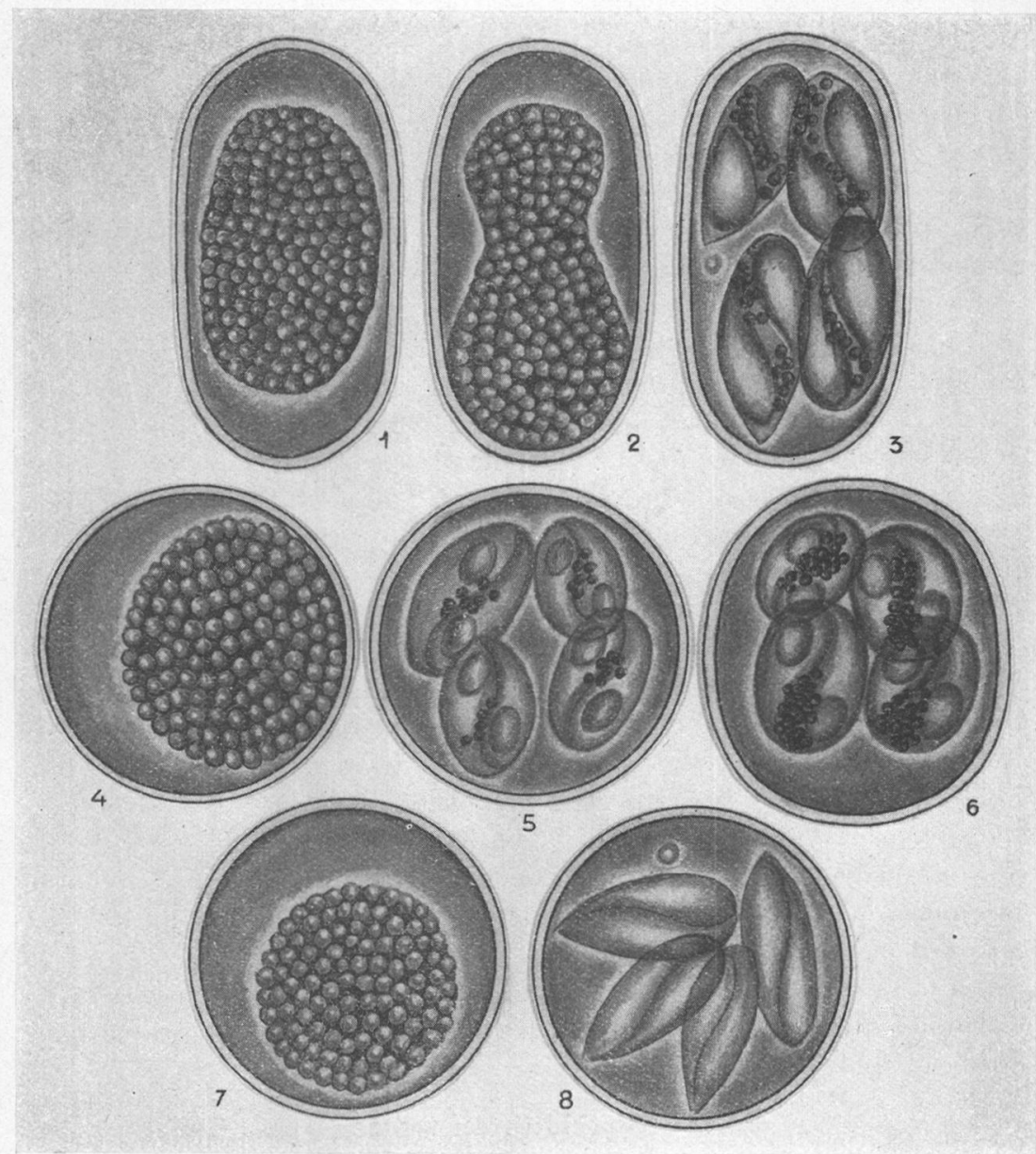

Fig. - 1 à 3 , Eimeria lyruri Galli-Valerio ; 4 et 6 , Eimeria brumpti n. sp. ; 7 et 8, Eimeria nadsoni n. sp.

(14,4-14,64 $\mu \times 5,4-9,88 \mu)$ contiennent deux sporozoïtes chacune. Dans l'oocyste il n'y a pas de reliquat, mais il existe un granule polaire.

Nous croyons que c'est la même coccidie que celle du coq de bruyère : Eimeria lyruri. 


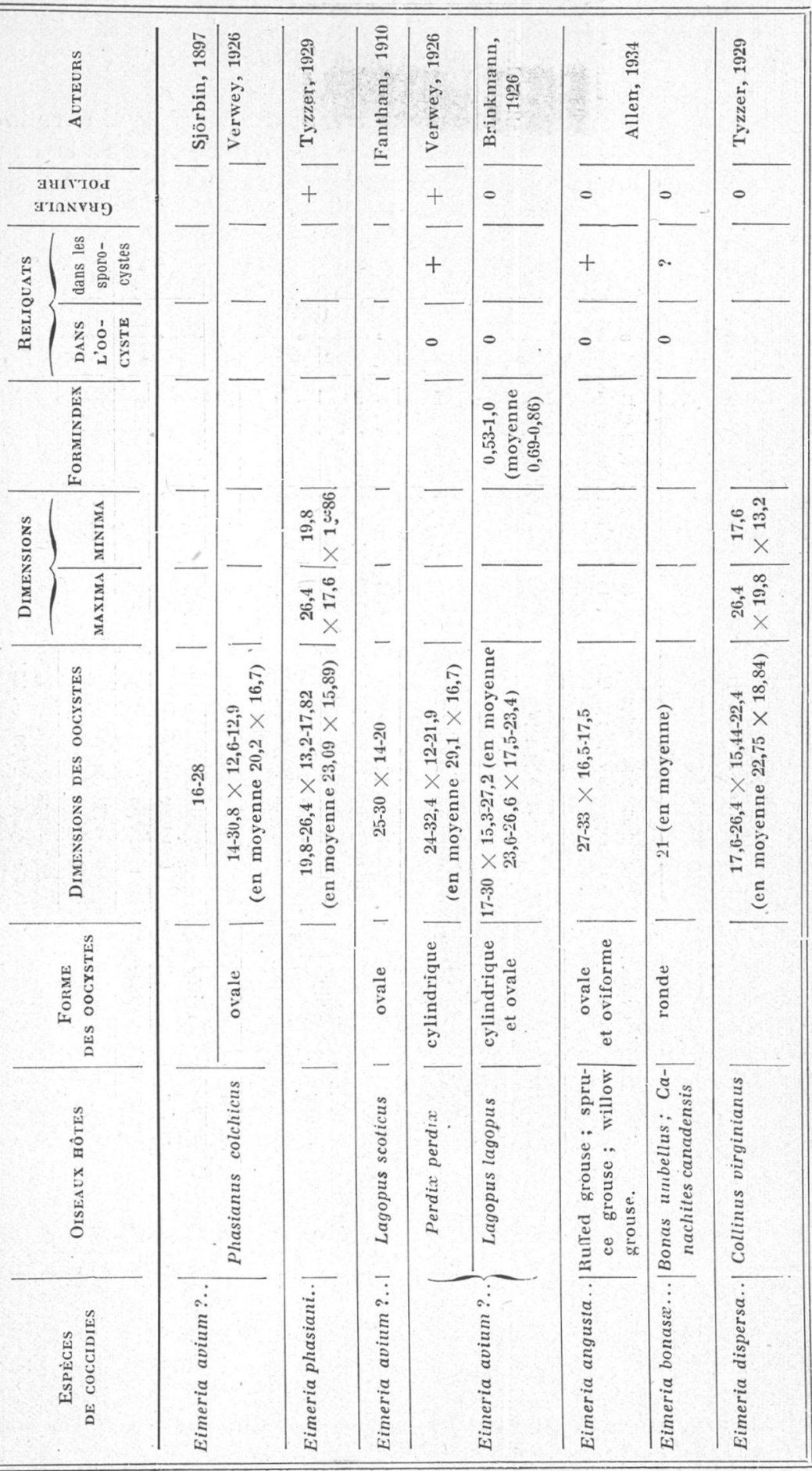




\begin{tabular}{|c|c|c|c|c|c|c|c|c|c|c|}
\hline 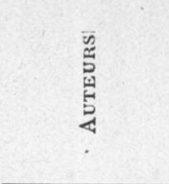 & 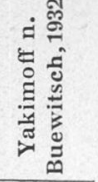 & 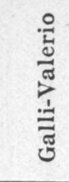 & & & 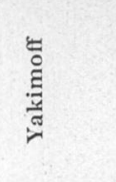 & $\Xi$ & & 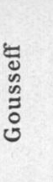 & & \\
\hline $\begin{array}{l}\text { a aiviod } \\
\text { 3таNvuy }\end{array}$ & + & & + & + & + & & \multicolumn{2}{|c|}{0} & \multicolumn{2}{|r|}{+} \\
\hline | & + & & + & a. & + & + & \multicolumn{2}{|c|}{+} & & a. \\
\hline 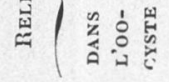 & 0 & & 0 & 0 & 0 & 0 & \multicolumn{2}{|c|}{0} & & 0 \\
\hline 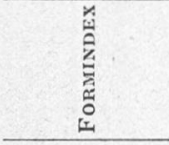 & 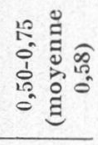 & & 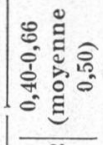 & 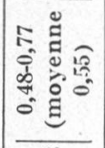 & 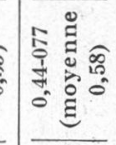 & - & & $\mid \begin{array}{l}0 \\
0 \\
0 \\
0 \\
0 \\
0 \\
0 \\
0\end{array}$ & & 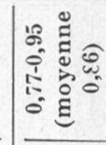 \\
\hline 旾 & भู & & 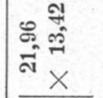 & न & 竎 & 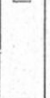 & فें & & $\begin{array}{l}\text { 을 } \\
\text { के }\end{array}$ & 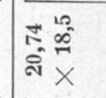 \\
\hline 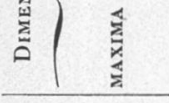 & 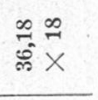 & & $\begin{array}{l}0 \\
0 \\
0 \\
0 \\
0 \\
0 \\
0 \\
\end{array}$ & 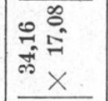 & 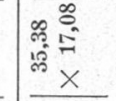 & - & in & & 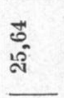 & 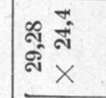 \\
\hline 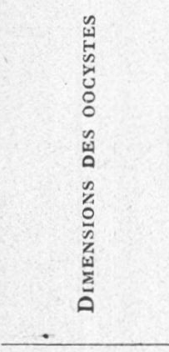 & 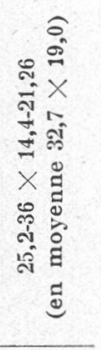 & 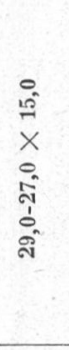 & 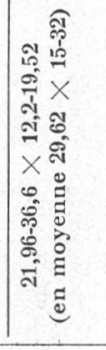 & 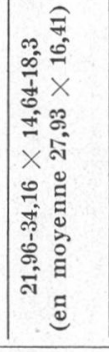 & 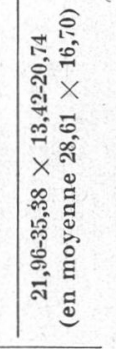 & 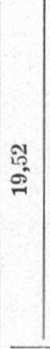 & 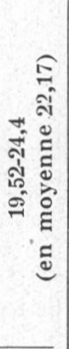 & 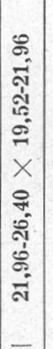 & 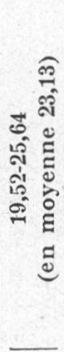 & 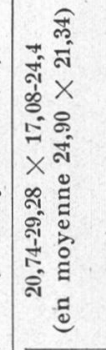 \\
\hline 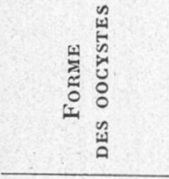 & 氙 & & 今. & $\frac{1}{\frac{1}{2}}$ & : & & : & 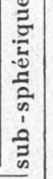 & : & 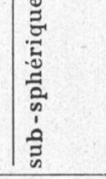 \\
\hline 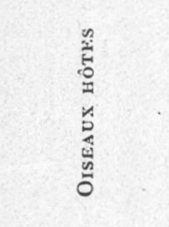 & 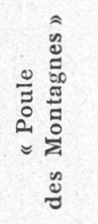 & & 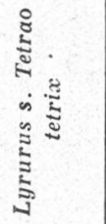 & 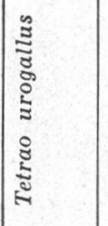 & 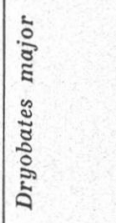 & 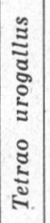 & 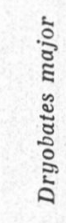 & & & 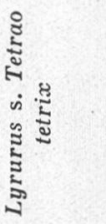 \\
\hline 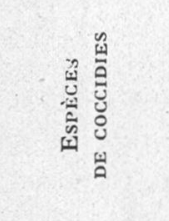 & 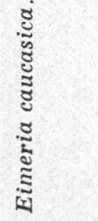 & & & 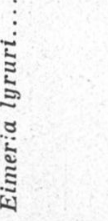 & & & 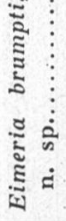 & & & 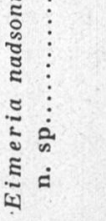 \\
\hline
\end{tabular}




\section{Deu xiÈme ESPÈce}

Cette coccidie se voit bien plus rarement que la précédente.

Les oocystes sont sphériques; ils mesurent 19,52 $\mu$ de diamètre. Quatre spores, mesurant $10,8 \mu \times 5,11 \mu$, se forment pendant la sporulation. Il n'y a ni reliquat, ni granule polaire dans l'oocyste, ce qui distingue cette coccidie de celle du coq de bruyère : Eimeria nadsoni.

Nous croyonș cette coccidie nouvelle et nous la nommons, en l'honneur du parasitologue français, le professeur E. Brumpt, Eimeria brumpti (nec Cauchemez !) n. sp.

\section{Coccidies de Dryobates major}

Nous examinâmes 16 Dryobates major, dont deux (un du district Polotzk et l'autre du district Gomel) hébergeaient des coccidies, appartenant à deux espèces.

\section{Première espèce}

Cette espèce a été trouvée dans le district de Gomel.

La forme des oocystes est sphérique et sub-sphérique. Leur couleur est jaunâtre; le protoplasme est excentrique. Les dimensions des formes sphériques, mesurées sur 75 oocystes, sont les suivantes: $19,52-24,40 \mu$, en moyenne $22,17 \mu$, le plus souvent $21,96 \mu$.

Les dimensions des formes sub-sphériques (ces formes sont plus rares) sont: $21,96-26,40 \mu \times 19,52-21,96 \mu$. Formindex : 1 : 0,81-0,94.

La sporulation donne quatre sporoblastes de $9,76 \mu$, où se forment des spores de 9,76-13,42 $\mu \times 6,10-7,32 \mu$. Ces dernières contiennent deux sporozoïtes. Il n'y a ni reliquat, ni granule polaire dans les oocystes, mais il existe un reliquat dans les sporocystes.

Il est possible que cette coccidie soit identique à l'Eimerie sphérique du tetras : Eimeria brumpti.

\section{DeuXième espèce}

Cette espèce a été trouvée dans le district de Polotzk.

La forme des oocystes est cylindrique. L'extrémité antérieure est quelquefois un peu plus étroite que l'extrémité postérieure. Plus rarement on rencontre des formes oviformes-oblongues. La membrane est jaunâtre, presque orange ; son épaisseur est de $1,22 \mu$. Il n'y a pas de micropyle.

Les dimensions, mesurées sur 83 oocystes, sont les suivantes : 21,96-35,38 $\mu \times 13,42-20,74 \mu$, en moyenne $28,61 \mu \times 16,70 \mu$, les plus grandes $35,38 \mu \times 17,08 \mu$, les plus petites $21,96 \mu \times 17,08 \mu$, 
le plus souvent $29,98 \mu \times 17,08 \mu$. Formindex : $1: 0,44-0,77$, en moyenne : $1: 0,58$, le plus souvent : $1: 0,58$.

Spores avec corpuscules de Stieda. Les dimensions des spores sont : $14,64-17,08 \mu \times 5,10-7,38 \mu$.

Seulement dans les sporocystes se trouve un reliquat. Les oocystes n'ont qu'un granule polaire.

En comparant cette coccidie à Eimeria lyruri du tétras et du coq de bruyère, nous croyons que fort probablement elle appartient à la même espèce.

De cette manière, les trois oiseaux, tétras, coq de bruyère et Dryobates major, hébergeaient trois espèces de coccidies, distribuées de la façon suivante :

\begin{tabular}{|c|c|c|c|}
\hline OISEAUX & E. lyruri & E. nadsoni & E. brumpti \\
\hline $\begin{array}{l}\text { Lyrurus tetrix s. Tetrao terix... } \\
\text { Tetrao urogallus.............. } \\
\text { Dryobates major.............. }\end{array}$ & $\begin{array}{l}+ \\
+ \\
+\end{array}$ & + & $\begin{array}{l}+ \\
+\end{array}$ \\
\hline
\end{tabular}

\section{Bibliographie}

Fantham (H. B.). - The morphology and life-hystory of Eimeria (Coccidium) avium; a sporozoan causing a fatal disease among young grouse. Proceed. Zoolog. Soc., London, XI, 1910, p. 672-691.

Gali-Valerio (B.). - Notes de Parasitologie et de technique parasitologique. Centralblatt $f$. Bakteriologie, CIII, 1927, p. 177-182.

- Notes de Parasitologie. Ibid., CXXII, 1929, p. 54-59.

Henry (D.). - Species of Coccidia in chickens and quail in California. Publications in Zoolog. of Univers. of California, XXXVI, 1931, $\mathbf{n}^{\circ} 9$, p. $157-70$.

LABbÉ (A.).. - Sur la morphologie et la classification des coccidies. C.R. Acad. Sciences, CXIX, 1894, p. 1019-1029.

SJörbing (N.). - Beiträge zur Kenntniss einiger Protozoen. Centralblatt $f$. Bakteriologie, XXII, 1897 , p. 675 .

Trzzer (E. E.). - Coccidosis in gallinaceous birds. Journal of Hyg., XI, $\mathrm{n}^{\circ} \mathbf{2}$, september 1929 .

Tyzzeri (E. E.), Theiler (H.) and Jones (E.). - Coccidiosis in gallinaceous birds. II. A comparativy study of the species of Eimeria of the chicken. Ibid., XV, 1932, p. 319-393.

YAkimofF (W. L.) und Buewitsch (B. I.). - Zur Frage der Coccidie wildlender Vögel in Azerbaidschan (Transkaukasus). Arch. f. Protistenkunde, LXXVII, 1932, p. 187-191.

Laboratoire de Parasitologie de l'Ecole vétérinaire de Léningrad et Service de Protozoologie de l'Institut scientifique vétérinaire d̀ Witebsk (Russie Blanche). 